J. Jpn. Soc. Comp. Statist., 7(1994), 89-103

\title{
ON SEQUENTIAL DESIGN BASED ON MARKOV CHAINS FOR SELECTING ONE OF TWO TREATMENTS IN CLINICAL TRIALS WITH DELAYED OBSERVATIONS
}

\author{
Hideyuki Douke*
}

\begin{abstract}
A truncated sequential design for selecting the better of two medical treatments is constructed by using a fundamental relation of Markov chains, with delayed observations based on a time lag of response time. The design is practical and applicable to a wide range of sequential clinical trials. The iterative formula of Markov chains is based on the trinomial random walk on a lattice diagram which adopts the untied and the tied pairs on the double-dichotomous responses. The study is to determine an optimum sequential design based on the evaluation of minimum expected loss.
\end{abstract}

\section{Introduction}

The truncated sequential procedures for selecting the better of two medical treatments are introduced by Armitage(1957) for testing the difference between the means of two binomial distributions. These procedures have been applied to sequential clinical trials for deciding the superiority between two treatments by using the double-dichotomous responses. These procedures only employ the untied pairs on the double-dichotomous responses such that each patient pair is tested sequentially by treatment $A$ and $B$, and then, each response of the patient is immediately available. However, in the practical clinical trials we must frequently deal with delayed observations where there exists a time lag between the administration of the treatment and the availability of the response. The statistical studies associated with the delayed observations are already presented by Anderson(1964), Choi and Clark(1970), Langenberg and Sirinivasan(1981). Anderson, Choi and Clark have dealt the probabilities of error associated with the terminal decision by using the delayed observations, after making a tentative decision. Langenberg and Sirinivasan have studied a fixed sample size plan for clinical trials with delayed observations.

Sequential procedures have been developed by modification of Wald's sequential probability ratio test, but our sequential designs are realized from minimizing the expected loss function based on Colton's criterion. Colton(1963) proposes that the only loss involved for attempting to design an optimal clinical trial is the consequence of being treated by the inferior among two treatments and that all other losses may be disregarded from the ethical point of view. On the other hand, Choi(1968) has presented a new type of sequential design for clinical trials by using a fundamental relation of Markov chains. Sugimura, Goto and Asano $(1969,1971)$ have already developed some types of open and closed optimum sequential

\footnotetext{
*Faculty of Engineering, Kyushu Tokai University, Kumamoto, 862, Japan.

Key words: Trinomial sequential design; Markov chains; Delayed observations
} 
designs based on Colton's decision theory which uses the fundamental equation of Markov chains without using delayed observations.

In the study we incorporate a sequential design based on Markov Chains for selecting one of two medical treatments in clinical trials by using delayed observation pairs. Here the procedure is constructed according to the following fundamental scheme.

(1) Firstly, during the trial period, a clinical trial will be performed sequentially on patient pairs with treatment $A$ on one member of the pair and treatment $B$ on the other. At a certain point in time, one of two treatments will be tentatively decided to be superior to the other according to a tentative decision rule.

(2) Secondly, immediately after the trial period, the better of the two treatments will be performed on all patient pairs arriving during the waiting period of $d$ unit times. At the end of the waiting period, the better treatment will be ultimately decided according to a final decision rule.

(3) Finally, based on the final decision rule, the treatment period will be performed as the better treatment for all the remaining patients.

Under the above mentioned scheme, we shall further decide an optimum statistical design utilizing an evaluation based on Colton's criterion. Since we deal with the untied and tied pairs on the responses, this is formulated by using general iterative formulas of Markov Chains, based on the trinomial random walk on a lattice diagram. Thus, we will consider the expected loss function, which is proportional to an average patient number of being treated with the inferior treatment. Then, our criterion will be to minimize the overall expected loss over a priori distribution of parameter.

An object of this study is to determine the optimum statistical design from the evaluation based on Colton's criterion and to compare the superiority between the following two sequential designs numerically. Specifically, the first design ( Design 1) will be constructed according to the above mentioned procedure and the second design (Design 2) will consist of the trial period and the treatment period without the waiting period. Thus, the second design is that the better of the two treatments will be decided at the end of the trial period. Based on results from the trial period, the treatment period will be performed as the better treatment for all the remaining patients.

\section{Assumptions}

The assumptions which pertrain throughout this paper are as follows:

Firstly, suppose a new treatment $A$ and standard treatment $B$ in clinical trials. Let $p_{A}$ be the probability of effectiveness with treatment $A$, and $p_{B}$ be of effectiveness with treatment $B$. Then we will consider the following hypotheses for comparing the superiority or inferiority of $A$ against $B$.

$$
\begin{gathered}
H_{2}: \delta=0 \text { against } H_{1}: \delta=\Delta(\Delta>0) \\
H_{2}: \delta=0 \text { against } H_{3}: \delta=-\Delta
\end{gathered}
$$

where $\delta=p_{A}-p_{B}$. Let $N$ be the total number of patients who are treated with one of two treatments, $A$ and $B$ for a disease, where $N$ is fixed and large. Next, a patient pair who arrives successively for treatment during the interval of unit time, will participate in the clinical trial by being administered $A$ on one patient and $B$ on the other in a patient pair allocated at random. Let $n$ be the number of allocated patient pairs. There is a time lag $d$ between the administration of the treatment and the availability of the response. Then, the result of effectiveness on a treatment is denoted by " 1 " and the result of ineffectiveness 


\section{DOUKE}

is denoted by " 0 ". If treatment $A$ is effectiveness and treatment $B$ is ineffectiveness, the delayed observation pair is denoted by $(1,0)$. The untied pairs on the double-dichotomous responses are denoted by $(1,0),(0,1)$, and tied pairs are denoted by $(1,1),(0,0)$. Thus the $m$ th observation pairs are denoted by one of four different pairs, $(1,0),(0,1),(1,1)$ and $(0,0)$. Let $m_{1}$ be the number of pairs $(1,0), m_{3}$ the number of pairs $(0,1)$, and $m_{2}$ the number of pairs $(1,1)$ and $(0,0)$ in the sequence obtained up to $m$-th observation pair. Next the set of three variables $\left\{m_{1}, m_{2}, m_{3}\right\}$ is distributed according to the trinomial distribution in which the number of trials is $m=\sum_{i=1}^{3} m_{i}$, and each probability is

$$
\begin{gathered}
p_{1}=P_{r}\{(a, b)=(1,0)\}=p_{A}\left(1-p_{B}\right), \\
p_{2}=P_{r}\{(a, b)=(1,1) \text { or }(0,0)\}=p_{A} p_{B}+\left(1-p_{A}\right)\left(1-p_{B}\right), \\
p_{3}=P_{r}\{(a, b)=(0,1)\}=\left(1-p_{A}\right) p_{B} .
\end{gathered}
$$

Here a parameter expressing a discrepancy of the effects among treatments $A$ and $B$ shall be given by $\delta$. Then, we can measure the superiority of effects among the two treatments by using a value of $\delta$. Namely, " $\delta>0$ " is equivalent to " $p_{A}>p_{B}$ ", $\delta=0$ " equivalent to " $p_{A}=p_{B}$ ", and " $\delta<0$ " equivalent to " $p_{A}<p_{B}$.

Secondly, we must assume that the only loss involved is the result of being treated with the inferior among two treatments and all other loss may be disregarded from the ethical point of view. Moreover, it is assumed that the cost is directly proportional to the true values of $|\delta|$. Namely, in terms of the expected loss formulation, if a patient is treated with the inferior treatment, a positive loss which is proportional to $|\delta|$ is scored for him.

Finally, we shall assume that a priori distribution $g(\delta)$ for the parameter $\delta$ which is assumed as a random variable instead of a priori two-dimensional distribution for two parameters $p_{1}$ and $p_{3}$ may be given as a probability distribution. It seems that the assumptions are reasonable in the practical clinical problem. In section 3, we shall also detail the following statistical designs in order to incorporate the procedure.

\section{Truncated sequential design 1}

3.1. Decision rules

The sequential design 1 for the equivalence test on (2.1) and (2.2) consists of trial period, waiting period and treatment period with the following decision rules. Namely, the design is constructed by the above mentioned fundamental scheme of procedure and the following stopping rules. Then we finally accept one of three hypotheses by use of delayed observation pairs, that is, treatment $A$ is superior to treatment $B, B$ is superior to $A$, and there is no difference between the two treatments. Here $M$ is the maximum time limit of trial period. Figure 3.1 illustrates the truncated sequential design 1 by coordinate representation, a number of allocated patient pair as the $n$ coordinate axis, and $y_{m}=m_{1}-m_{3}$ as the $y_{m}$ coordinate axis, where $n=m+d$. The fixed upper and lower boundary lines for accepting $H_{1}$ and $H_{3}$ are represented on the graph in Figure 3.1., $U\left(y_{m}=c\right)$ and $L\left(y_{m}=-c\right)$ respectively. The middle boundary line for accepting $H_{2}$ takes the form of a virtual line at $M$, where $d \geq 2 c$ and $M \geq d+c$ are the necessary relations to be mentioned later. Figure 3.1 shows that when a sequence is given as $\{(1,0),(0,1),(1,0),(1,0),(0,0),(1,0)\}$ with $d=6$ under $c=3$ and $M=20$, a point of 1 -st observation pair $(1,0)$ is plotted as the coordinates $\left(n, y_{m}\right)=(7,1)$, and the other points of the sequence are plotted successively in the same way. Then the sequence reaches the upper boundary first in Figure 3.1, thus the tentative decision $H_{1}$ is accepted. 
We give more detail in the next sequential procedure by adopting tentative and final decision rules. The procedure is classified from the following cases, that is, case 1-1 and case 1-2 ( see Figure 3.1) are that the tentative decision is carried out before the $(M-d)$-th observation pair is observed, and case 2 ( see Figure 3.2) is that the tentative decision is carried out just at the $(M-d)$-th observation pair without previously reaching both boundaries.

\section{( Case 1-1)}

If a sequence reaches the upper boundary at the $m$-th discrete step corresponding to $m$-th observation pair, where $c \leq m \leq M-d$, (i.e., if $m_{1}-m_{3}=c$ firstly at the $m$-th step, where $m=m_{1}+m_{2}+m_{3}, m_{1}$ is the number of $(1,0)$ pairs, $m_{2}$ is the number of $(1,1)$ or $(0,0)$ pairs, and $m_{3}$ is the number of $(0,1)$ pairs), then one accepts the tentative decision $H_{1}$ at the end of the trial period, and $d$ patients pairs arriving during the waiting period are assigned to $A$ treatment. Here we give the final decisions as follows:

(1) now, let $d_{1}$ be the number of $(1,0)$ pairs, and $d_{3}$ be of $(0,1)$ pairs, and $d_{2}$ be of $(0,0)$ or $(1,1)$ during the waiting period, $d=d_{1}+d_{2}+d_{3}$, if $d_{1}-d_{3} \geq 0$, then assign $A$ for the remaining patients $N-2 m-4 d$ during the treatment period, as one accepts the terminal decision $H_{1}$,

(2) if $0>d_{1}-d_{3}>-2 c$, then assign treatment $A$ to half of the remaining patients $N-2 m-4 d$ and treatment $B$ to the other half, as the terminal decision $\mathrm{H}_{2}$,

(3) if $d_{1}-d_{3} \leq-2 c$, then assign $B$ for the remaining patients $N-2 m-4 d$, as the terminal decision $\mathrm{H}_{3}$, where $d \geq 2 c$.

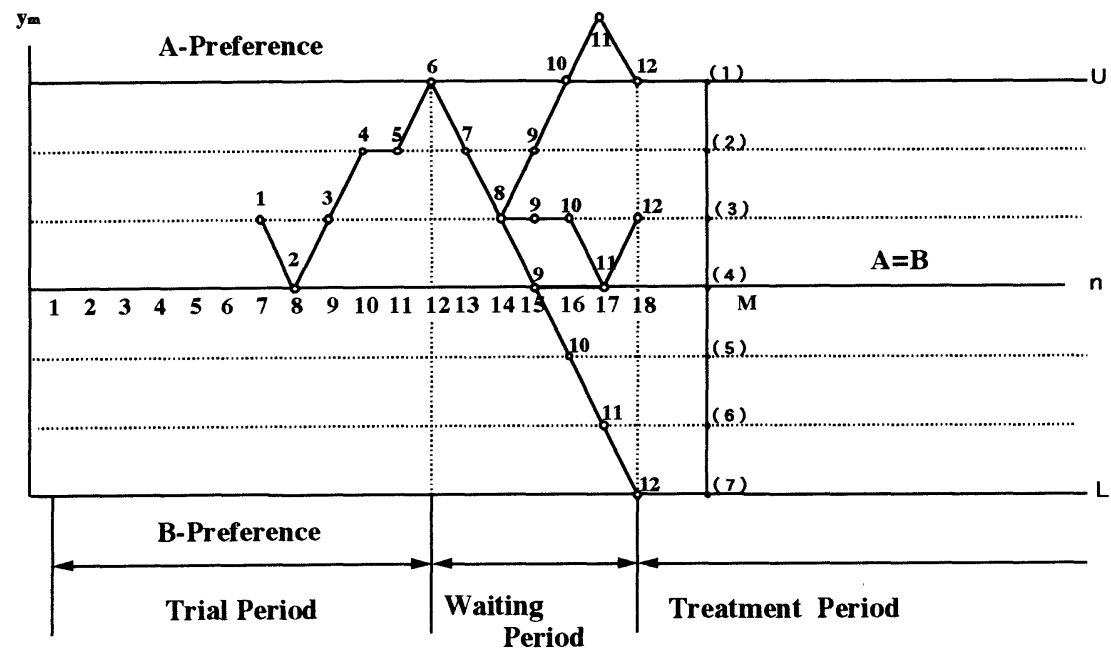

Figure 3.1 Sequential design 1 for Case 1-1 $(M=20, c=3, d=6)$ 


\section{DOUKE}

( Case 1-2)

If a sequence reaches the lower boundary at the $m$-th step, where $c \leq m \leq M-d$, then one accepts the tentative decision $H_{3}$ and $d$ patients pairs arriving during the waiting period are assigned to $B$ treatment. Moreover the terminal decisions are as follows:

(1) if $d_{1}-d_{3} \leq 0$ at the end of the waiting period, then assign $B$ for the remaining patients $N-2 m-4 d$, as one accepts the terminal decision $H_{3}$,

(2) if $0<d_{1}-d_{3}<2 c$, then assign treatment $A$ at half of the remaining patients $N-2 m-4 d$ and treatment $B$ at the other half, as the terminal decision $\mathrm{H}_{2}$,

(3) if $d_{1}-d_{3} \geq 2 c$, then assign $A$ for the remaining patients $N-2 m-4 d$, as the terminal decision $H_{1}$, where $d \geq 2 c$.

\section{( Case 2)}

There are the path points $l, l=2, \cdots, 2 c$, which are the ordinate value of the lattice points for accepting $H_{2}$ at $(M-d)$-th observation pair, denoted by $(2),(3), \cdots,(6)$ in case of Figure 3.2. If a sequence reaches neither the upper or lower boundary before the $(M-d)$-th observation pair is observed, and arrives at the $l$-th path point at the $(M-d)$-th step, (i.e., if $c>m_{1}-m_{3}>-c$ at the $(M-d)$-th step without previously reaching both boundaries ), then one accepts the tentative decision $H_{2}$ at the end of the trial period, and assigns treatment $A$ to half of the patient pairs $d$ arriving during the waiting period and treatment $B$ to the other half.

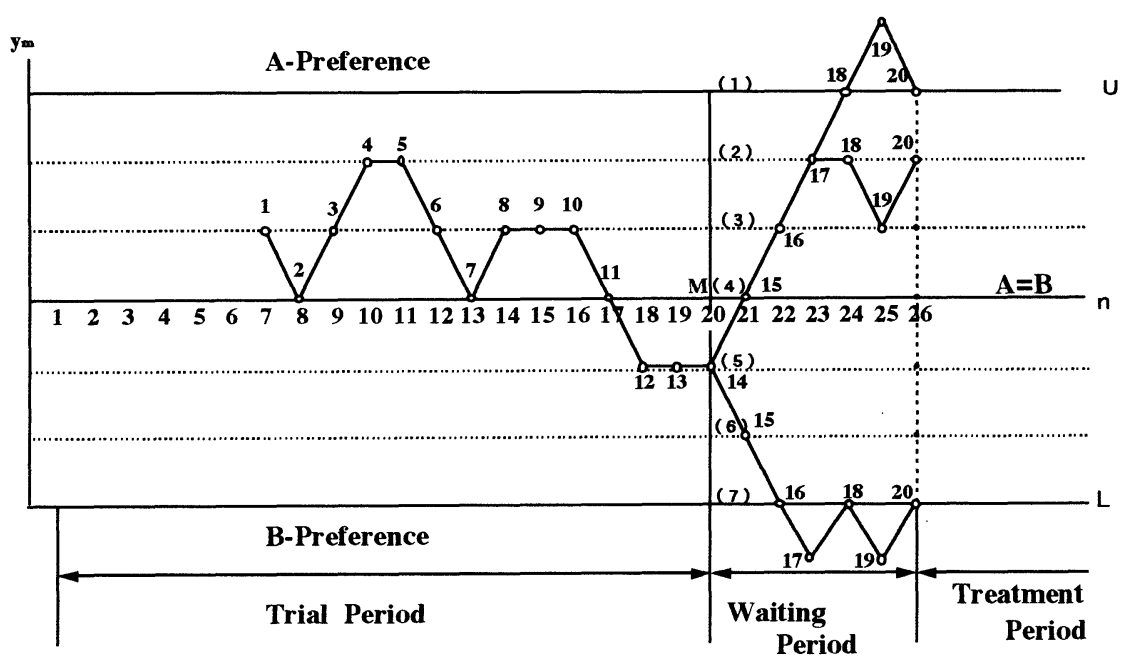

Figure 3.2 Sequential design 1 for Case $2 \quad(M=20, c=3, d=6)$

Here the terminal decisions are as follows:

(1) if $d_{1}-d_{3} \geq l-1$ at the end of the waiting period, then assign $A$ for the remaining patients $N-2 M-2 d$ during the treatment period, as one accepts the terminal decision $H_{1}$, 
(2) if $l-1>d_{1}-d_{3}>l-(2 c+1)$, then assign treatment $A$ to half of the remaining patients $N-2 M-2 d$ and treatment $B$ to the other half, as the terminal decision $\mathrm{H}_{2}$,

(3) if $d_{3}-d_{1} \geq(2 c+1)-l$, then assign $B$ for remaining patients $N-2 M-2 d$, as the terminal decision $\bar{H}_{3}$.

\subsection{Construction of overall expected loss function}

The sequential designs based on the above mentioned decision rules are characterized by the probabilities of tentatively accepting one of the three hypotheses of $H_{i}, \alpha_{i}, i=1,2,3$, the conditional probabilities that one accepts the terminal decision $H_{j}$, given that one accepts the tentative decision $H_{i}$, are $\alpha_{i j}, i=1,3, j=1,2,3$, and the joint probabilities that one accepts the tentative decision $H_{2}$ and accepts the terminal decision $H_{j}$, are $\alpha^{2, j}, j=1,2,3$.

Here $y_{m}$ becomes a trinomial random walk on a lattice diagram. And the absorption probabilities where a sequence crosses upper, lower and middle boundary point, can be calculated exactly by using the general iterative formulas of Markov Chains. Then we shall show the average patient pair number and the over all expected loss function by the following way.

Let $\mathbf{s}_{0}\left(p_{1}, p_{3}\right)=\left(s_{1}, s_{2}, \cdots, s_{l}, \cdots, s_{2 c+1}\right)$ be a $(2 c+1)$ dimensional row vector which arranges the initial probabilities, where the $l$-th component $s_{l}, l=1,2, \cdots, 2 c+1$, is the probability of reaching the $l$-th path point at the $c$-th step. It follows that for each

$$
s_{l}=\sum_{\left(m_{1}, m_{2}, m_{3}\right)} c ! / m_{1} ! m_{2} ! m_{3} ! \cdot p_{1}^{m_{1}} p_{2}^{m_{2}} p_{3}^{m_{3}}
$$

where the symbol $\sum$ denotes the summation of all combinations $\left(m_{1}, m_{2}, m_{3}\right)$ of three integers $m_{1}, m_{2}$ and $m_{3}$ which satisfy three conditions

$$
\sum_{i=1}^{3} i m_{i}=c+l-1, \sum_{i=1}^{3} m_{i}=c, c \geq m_{1}, m_{2}, m_{3} \geq 0
$$

for the fixed values of $p_{1}, p_{3}$ and $c$. It should be noted that the sum of all components $s_{l}$ in $\mathbf{s}_{0}\left(p_{1}, p_{3}\right)$ is equal to the expansion formula of $\left(p_{1}+p_{2}+p_{3}\right)^{c}$, where $\sum_{l=1}^{2 c+1} s_{l}=1$.

Here, $T\left(p_{1}, p_{3}\right)$ is the $(2 c+1) \times(2 c+1)$ transition probability matrix from each path point at the $(c+j)-$ th step to these points at the $(c+j+1)-$ th $j=0,1, \cdots, M-d-c-1$ as,

$$
T\left(p_{1}, p_{3}\right)=\left[\begin{array}{lllllll}
1 & 0 & \cdots & \cdots & \cdots & \cdots & 0 \\
p_{1} & p_{2} & p_{3} & 0 & \cdots & \cdots & 0 \\
0 & p_{1} & p_{2} & p_{3} & 0 & \cdots & 0 \\
\cdot & & & & & & \cdot \\
\cdot & & & & & & \cdot \\
. & \cdots & \cdots & \cdots & \cdots & \cdots & \cdot \\
. & & & & & & \cdot \\
. & & & & & & \cdot \\
0 & \cdots & \cdots & p_{1} & p_{2} & p_{3} & 0 \\
0 & \cdots & \cdots & \cdots & p_{1} & p_{2} & p_{3} \\
0 & \cdots & \cdots & \cdots & \cdots & 0 & 1
\end{array}\right]
$$




\section{DOUKE}

Let $\mathbf{s}_{j}\left(p_{1}, p_{3}\right)=\left(s_{1}^{j}, s_{2}^{j}, \cdots, s_{i}^{j}, \cdots, s_{2 c+1}^{j}\right)$ be a $(2 c+1)$ dimensional vector, where the $i$-th component represents the probability of reaching the $i$-th path point at the $(c+j)$-th step $(i=1,2, \cdots, 2 c+1 ; j=0,1, \cdots, M-d-c)$. Then, by a fundamental relation of Markov chains,

$$
\mathbf{s}_{j}\left(p_{1}, p_{3}\right)=\mathbf{s}_{0}\left(p_{1}, p_{3}\right) \cdot T^{j}\left(p_{1}, p_{3}\right)
$$

where $T^{0}\left(p_{1}, p_{3}\right)$ is defined as a $(2 c+1) \times(2 c+1)$ unit matrix. Let $\mathbf{e}_{\boldsymbol{i}}$ be a $(2 c+1)$ dimensional row vectors such that the $i$-th component is 1 and the others are all zeros, that is,

$$
\mathbf{e}_{i}=(0, \cdots, 0,1,0, \cdots, 0), \quad i=1,2, \cdots, 2 c+1
$$

Since the $1-$ st and $(2 c+1)$-th path point are absorbing barriers, it can be shown that

$$
\begin{aligned}
\alpha_{1}^{j} & =P_{r}\left\{\text { accepting tentatively } A \text { until }(c+j)-\text { th step } \mid p_{1}, p_{3} ; c, M, d\right\} \\
& =\mathbf{s}_{0} \cdot T^{j} \cdot \mathbf{e}_{1}^{\prime}=\mathbf{s}_{j}\left(p_{1}, p_{3}\right) \cdot \mathbf{e}^{\prime}{ }_{1}, \quad j=0,1,2, \cdots, h,
\end{aligned}
$$

where $\mathbf{s}_{0}=\mathbf{s}_{0}\left(p_{1}, p_{3}\right), T=T\left(p_{1}, p_{3}\right)$ and $h=M-c-d$.

$$
\begin{aligned}
\alpha_{3}^{j} & =P_{r}\left\{\text { accepting tentatively } B \text { until }(c+j)-\text { th step } \mid p_{1}, p_{3} ; c, M, d\right\} \\
& =\mathbf{s}_{j}\left(p_{1}, p_{3}\right) \cdot \mathbf{e}^{\prime}{ }_{2 c+1}, \quad j=0,1,2, \cdots, h,
\end{aligned}
$$

Then, $\alpha_{i}, i=1,2,3$, is denoted as,

$$
\alpha_{1}=\alpha_{1}^{h}, \alpha_{3}=\alpha_{3}^{h}, \alpha_{2}=1-\alpha_{1}-\alpha_{3} .
$$

The conditional probabilities that one accepts the terminal decision $H_{j}$, given that one accepts the tentative decision $H_{i}$, are $\alpha_{i j}, i=1,3, j=1,2,3$.

$$
\begin{aligned}
\alpha_{11} & =P_{r}\left\{\text { accepting } H_{1} \text { finally } \mid \text { acceptance of } H_{1} \text { tentatively }\right\} \\
& =\Sigma_{1} d ! / d_{1} ! d_{2} ! d_{3} ! \cdot p_{1}^{d_{1}} p_{2}^{d_{2}} p_{3}^{d_{3}}
\end{aligned}
$$

where the notation $\Sigma_{1}$ represents the summation overall triples $\left(d_{1}, d_{2}, d_{3}\right)$ under the condition $d_{1} \geq d_{3}$ and $d_{1}+d_{2}+d_{3}=d$.

$$
\begin{aligned}
\alpha_{12} & =P_{r}\left\{\text { accepting } H_{2} \text { finally } \mid \text { acceptance of } H_{1} \text { tentatively }\right\} \\
& =\Sigma_{2} d ! / d_{1} ! d_{2} ! d_{3} ! \cdot p_{1}^{d_{1}} p_{2}^{d_{2}} p_{3}^{d_{3}}
\end{aligned}
$$

where the notation $\Sigma_{2}$ represents the summation overall triples $\left(d_{1}, d_{2}, d_{3}\right)$ under $0<d_{3}-$ $d_{1}<2 c$ and $d_{1}+d_{2}+d_{3}=d$.

$$
\begin{aligned}
\alpha_{13} & =P_{r}\left\{\text { accepting } H_{3} \text { finally } \mid \text { acceptance of } H_{1} \text { tentatively }\right\} \\
& =\Sigma_{3} d ! / d_{1} ! d_{2} ! d_{3} ! \cdot p_{1}^{d_{1}} p_{2}^{d_{2}} p_{3}^{d_{3}}
\end{aligned}
$$

where the notation $\Sigma_{3}$ represents the summation overall triples $\left(d_{1}, d_{2}, d_{3}\right)$ under $d_{3}-d_{1} \geq 2 c$ and $d_{1}+d_{2}+d_{3}=d$. Now, $\alpha_{31}=\alpha_{13}^{*}$, where $\alpha_{13}^{*}$ represents the function obtained by 
exchanging $p_{1}$ and $p_{3}$ at $\alpha_{13}$. Similarly, $\alpha_{32}=\alpha_{12}^{*}$, and $\alpha_{33}=\alpha_{11}^{*}$, where $\alpha_{12}^{*}$ and $\alpha_{11}^{*}$ represent respectively the function obtained by exchanging $p_{1}$ and $p_{3}$ at $\alpha_{12}$, and at $\alpha_{11}$.

The joint probabilities that one accepts the tentative decision $H_{2}$ and accepts the tentative decision $H_{j}, j=1,2,3$, are $\alpha^{2, j}, j=1,2,3$.

$$
\alpha^{21}=\Sigma_{l=2}^{2 c} \Sigma_{4} d ! / d_{1} ! d_{2} ! d_{3} ! \cdot p_{1}^{d_{1}} p_{2}^{d_{2}} p_{3}^{d_{3}} \cdot \mathbf{s}_{h}\left(p_{1}, p_{3}\right) \cdot \mathbf{e}_{l}^{\prime},
$$

where second summation $\Sigma_{4}$ is over $\left\{d_{1}-d_{3} \geq l-1, d_{1}+d_{2}+d_{3}=d\right\}$.

$$
\alpha^{22}=\Sigma_{l=2}^{2 c} \Sigma_{5} d ! / d_{1} ! d_{2} ! d_{3} ! \cdot p_{1}^{d_{1}} p_{2}^{d_{2}} p_{3}^{d_{3}} \cdot \mathbf{s}_{h}\left(p_{1}, p_{3}\right) \cdot \mathbf{e}_{l}^{\prime},
$$

where second summation $\Sigma_{5}$ is over $\left\{l-1>d_{1}-d_{3}>l-(2 c+1), d_{1}+d_{2}+d_{3}=d\right\}$.

$$
\alpha^{23}=\Sigma_{l=2}^{2 c} \Sigma_{6} d ! / d_{1} ! d_{2} ! d_{3} ! \cdot p_{1}^{d_{1}} p_{2}^{d_{2}} p_{3}^{d_{3}} \cdot \mathbf{s}_{h}\left(p_{1}, p_{3}\right) \cdot \mathbf{e}_{l}^{\prime},
$$

where second summation $\Sigma_{6}$ is over $\left\{d_{3}-d_{1} \geq(2 c+1)-l, d_{1}+d_{2}+d_{3}=d\right\}$.

Let $E\left(n \mid p_{1}, p_{3} ; M, c, d\right)$ be the average patient pair number during the trial period. $E\left(n \mid p_{1}, p_{3} ; M, c, d\right)$ can be obtained by the following equation,

$$
\begin{aligned}
E(n)= & (d+c)\left(\alpha_{1}^{0}+\alpha_{3}^{0}\right)+(d+c+1)\left(\alpha_{1}^{1}-\alpha_{1}^{0}+\alpha_{3}^{1}-\alpha_{3}^{0}\right) \\
& +(d+c+2)\left(\alpha_{1}^{2}-\alpha_{1}^{1}+\alpha_{3}^{2}-\alpha_{3}^{1}\right) \\
& +\cdots+(d+c+h)\left(\alpha_{1}^{h}-\alpha_{1}^{h-1}+\alpha_{3}^{h}-\alpha_{3}^{h-1}\right)+M \alpha_{2} \\
= & (d+c) \mathbf{s}_{0} \mathbf{e}^{\prime}+(d+c+1)\left(\mathbf{s}_{1}-\mathbf{s}_{0}\right) \mathbf{e}^{\prime} \\
& +(d+c+2)\left(\mathbf{s}_{2}-\mathbf{s}_{1}\right) \mathbf{e}^{\prime} \\
& +\cdots+M\left(\mathbf{s}_{h}-\mathbf{s}_{h-1}\right) \mathbf{e}^{\prime} \\
& +M\left\{1-\mathbf{s}_{h} \mathbf{e}^{\prime}\right\} \\
= & M-\sum_{k=0}^{h-1} \mathbf{s}_{k} \mathbf{e}^{\prime}
\end{aligned}
$$

where $\mathbf{e}=\mathbf{e}_{1}+\mathbf{e}_{2 c+1}$. If treatment $A$ is inferior to treatment $B$, then the expected loss function due to performing the treatment $A$, denoted by $[E L o s s]_{A}$, is obtained from the assumption in $\S 2$ as follows,

$$
\begin{aligned}
{[E \text { Loss }]_{A} } & =|\delta| G[E(n) \\
& +2 d \alpha_{1}+\alpha_{1}\left\{(N-2 E(n)-2 d) \alpha_{11}+(N / 2-E(n)-d) \alpha_{12}\right\} \\
& +\alpha_{3}\left\{(N-2 E(n)-2 d) \alpha_{31}+(N / 2-E(n)-d) \alpha_{32}\right\} \\
& \left.+d \alpha_{2}+(N-2 M-2 d) \alpha^{21}+(N / 2-M-d) \alpha^{22}\right] .
\end{aligned}
$$

where $G$ is a constant of positive proportionality. In the same manner, if treatment $B$ is inferior to treatment $A$, then the expected loss function due to performing the treatment $B$, denoted by $[E L o s s]_{B}$, is obtained as follows, 


\section{DOUKE}

$$
\begin{aligned}
{[E \text { Loss }]_{B} } & =|\delta| G[E(n) \\
& +2 d \alpha_{3}+\alpha_{3}\left\{(N-2 E(n)-2 d) \alpha_{33}+(N / 2-E(n)-d) \alpha_{32}\right\} \\
& +\alpha_{1}\left\{(N-2 E(n)-2 d) \alpha_{13}+(N / 2-E(n)-d) \alpha_{12}\right\} \\
& \left.+d \alpha_{2}+(N-2 M-2 d) \alpha^{23}+(N / 2-M-d) \alpha^{22}\right] .
\end{aligned}
$$

By integration (3.9) and (3.10) over a priori distribution for $\delta$, the over all expected loss function (i.e., the average risk ), denoted by $\overline{E L o s s} / N G$, is then constructed.

$$
\overline{E L o s s} / N G=\left\{\int_{-1}^{0}[E L o s s]_{A} g(\delta) d \delta+\int_{0}^{1}[E L o s s]_{B} g(\delta) d \delta\right\} / N G,
$$

where $g(\delta)$ denotes the probability density function of a priori distribution for the parameter $\delta$. If $g(\delta)$ is a discrete probability function, the above integrations are replaced by summations in the following way:

$$
\overline{E L o s s} / N G=\left\{\sum_{\left\{i \mid-1 \leq \delta_{i}<0\right\}}[E L o s s]_{A} g\left(\delta_{i}\right)+\sum_{\left\{i \mid 0<\delta_{i} \leq 1\right\}}[E L o s s]_{B} g\left(\delta_{i}\right)\right\} / N G .
$$

\section{Truncated sequential design 2}

The sequential design 2 for the equivalence test on (2.1) and (2.2) is constructed by trial period and treatment period with the following decision rules. Namely, the procedure is that the treatment period is performed as the better treatment for all the remaining patients, after the better treatment is decided at the end of trial period. The design is incorporated in a similar manner to the sequential design 1, and is shown in Figure 4.1. The truncated sequential procedure is given as follows.

\section{( Case 1-1)}

If a sequence reaches the upper boundary at the $m$-th step, where $c \leq m \leq M-d$, as one accepts the decision $H_{1}$, then assign $A$ for the remaining patients $N-2 m-2 d$ during the treatment period.

( Case 1-2)

If a sequence reaches the lower boundary at the $m$-th step, where $c \leq m \leq M-d$, as one accepts the decision $H_{3}$, then assign $B$ for the remaining patients $N-2 m-2 d$.

( Case 2)

If a sequence reaches neither the upper or the lower boundary before the $(M-d)$-th observation pair is observed, as one accepts the decision $H_{2}$, then assign treatment $A$ to half of the remaining patients $(N-2 M) / 2$ and treatment $B$ to the other half. 


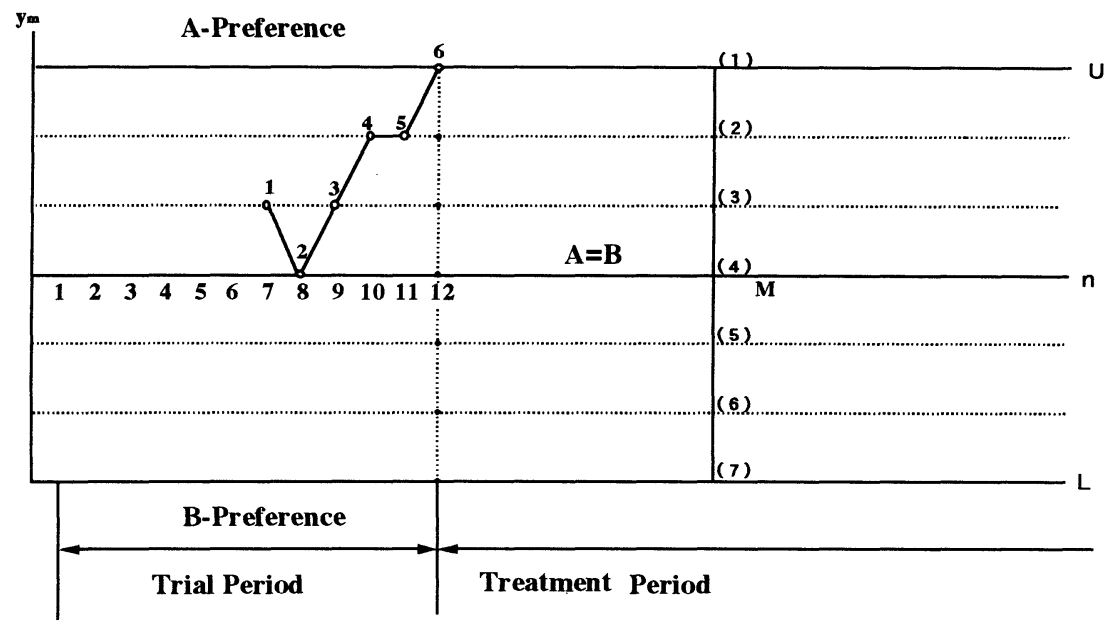

Figure 4.1 Sequential design 2 for Case 1-1 $(M=20, c=3, d=6)$

Three probabilities of accepting $H_{1}, H_{2}$ and $H_{3}, \alpha_{i}, i=1,2,3$, and the average patient pair number during the trial period, $E(n)$, equal to those of design 1 . The expected loss functions are,

$$
[E L o s s]_{A}=|\delta| G\left[E(n)+\alpha_{1}(N-2 E(n))+\alpha_{2}(N / 2-M)\right]
$$

and

$$
[E L o s s]_{B}=|\delta| G\left[E(n)+\alpha_{3}(N-2 E(n))+\alpha_{2}(N / 2-M)\right] .
$$

The overall expected loss function, $\overline{E \text { Loss }} / N G$, has the same form of (3.11)(or (3.12)). The object of statistical design is to determine an optimum value $c$ which minimizes the overall expected loss function for each value of $d$ under $N, M, d, \delta, g(\delta)$ given.

\section{Numerical Examples}

We shall deal with some numerical examples in the various types of pair $\left(p_{A}, p_{B}\right)$ with a priori distribution $g(\delta)$ in Table 5.1. To investigate the values of $\overline{E L o s s} / N G$ for each of $d$, Table 5.2 and Table 5.3 show respectively the values of $\overline{E L o s s} / N G$ with $c$ for each of value $d$ on Type 3 under $M=30$ and $N=1000$ on design 1 and design 2 . Here the optimum value of $\overline{E L o s s} / N G$ for each of $d$ is denoted by $E L^{*}$ and the value of $c$ corresponding to $E L^{*}$ is denoted by $c^{*}$. The results are shown as follows:

(1) The values of $c^{*}$ take $c=2,3$ for design 1 and design 2 .

(2) The value of $E L^{*}$ on design 2 has smaller value than the value of $E L^{*}$ on design 1 for each of value $d$ when $d \leq 8$, but the value of $E L^{*}$ on design 1 has smaller value than it on design 2 when $d \geq 10$.

Then, we can see that the other type of $\left(p_{A}, p_{B}\right)$ has a similar result. To compare the results for each of the types on design 1 and design 2, Table 5.4 and Table 5.5 show the 
probabilities $\overline{\alpha_{i}}$ of accepting finally the hypotheses of $H_{i}, E L^{*}$ and $c^{*}$ for $d=18$. Here $\overline{\alpha_{i}}=\alpha_{1} \alpha_{1 i}+\alpha^{2 i}+\alpha_{3} \alpha_{3 i}, i=1,2,3$ on design 1 , and $\overline{\alpha_{i}}=\alpha_{i}, i=1,2,3$ on design 2 .

In Table 5.4 and Table 5.5, $\overline{\alpha_{1}}$ for any $\left(p_{A}, p_{B}\right)$ takes a large value except $\left(p_{A}, p_{B}\right)=$ $(0.7,0.6)$. Moreover Type 1 has lower values of $E L^{*}$, because each of $\delta$ on Type 1 takes a large value, while Type 3 has higher values of $E L^{*}$, because each of $\delta$ on Type 3 takes a small value.

Table 5.6 gives the relation between $\overline{\alpha_{1}}$ and $M$ for each of three types under $N=$ $1000, d=18$ on design 1. Furthermore, Figure 5.1 shows the comparison of curve of $\overline{\alpha_{1}}$ for the selected $\left(p_{A}, p_{B}\right)$ in each of three types, i.e., $\left(p_{A}, p_{B}\right)=(0.7,0.4)$ on Type $1,\left(p_{A}, p_{B}\right)=$ $(0.7,0.5)$ on Type 2 and $\left(p_{A}, p_{B}\right)=(0.7,0.6)$ on Type 3 . It becomes clear as follows:

(3) The curve for Type 3 increases as $M$ increases, while the curve for Type 1 is almost flat.

Table 5.7 and Table 5.8 give the relation between $E L^{*}$ and $M$ for the above three types on design 1 and design 2 under $N=1000, d=18$, and Figure 5.2 shows the comparison of curve of $E L^{*}$ among three types. It is mentioned as follows:

(4) As to design 1 , the curve of $E L^{*}$ for Type 3 decreases as $M$ increases, however the curve for Type 1 is almost unchangeable as $M$ increases. As to design 2, the curves of $E L^{*}$ for all Types decrease monotonously.

(5) As to Type 2 and Type 3, the value of $E L^{*}$ on design 1 has a smaller value than the value of $E L^{*}$ on design 2 for each of value $M$ when $M \leq 50$, but the value of $E L^{*}$ on design 2 has a smaller value than the value of $E L^{*}$ on design 1 when $M \geq 60$.

Table 5.9 and Table 5.10 give the relation between $E L^{*}$ and $N$ for the above three types on design 1 and design 2 under $M=30, d=18$. It is mentioned as follows:

(6) The values of $E L^{*}$ increase as $N$ increases, specially the value on design 2

increases remarkably, because $E L^{*}$ is influenced by the design without waiting period.

From the above results, it becomes clear that the superiority among two designs depends on the values of $M$ and $d$.

\section{Further discussions}

The study is concerned with two sequential designs for selecting one of two treatments with delayed observations in clinical trials. In practice, when an analyzer wishs to use the sequential designs, the procedure is as follows:

(1) The probabilities $\left(p_{A}, p_{B}\right)$, a priori distribution $g(\delta)$ and the time lag $d$ should be set up by preliminary trials and clinical knowledge.

(2) The total number of patients $N$ must be estimated from a preliminary survey by restricting region and period.

(3) We can find out the value of $M$ corresponding to $\overline{\alpha_{i}}$ to be satisfied by the specified error probability in the Table which shows the relation between $M$ and $\overline{\alpha_{i}}$.

(4) The values of $E L^{*}$ between design 1 and design 2 are compared under the given $M$, the better of two designs can be selected and the value of $c^{*}$ is obtained.

(5) Thus the analyzer can use the sequential design based on observation pairs under $M$ and $c^{*}$ obtained.

However, regarding use of the design, there are still several difficulties as follows:

(6) In this study, the time lag $d$ is taken by some fixed values, however if the 
time lag will show a variation by the differences among individuals from practical and clinical aspects, we should deal with it as a random variable.

(7) When $M$ is chosen in terms of the precision of test, the average patient pair number $E(n)$ often takes a large value. Then we will again set up an adequate value $M$ from the economical point of view.

(8) It is difficult to decide the total number $N$ of patients on a disease in clinical trials, so we will suggest estimating the value of $N$ from a sample survey.

\section{Acknowledgments}

The author wishes to express his gratitude to Professor Masahiko Sugimura of Kyushu Tokai University for his kind guidance and valuable suggestions on this paper. He is also deeply grateful to the referees for their comments and suggestions.

Table 5.1 The types for each $\left(p_{A}, p_{B}\right)$

\begin{tabular}{|c|c|c|c|c|c|c|c|c|c|c|c|c|c|c|c|}
\hline$p_{B}$ & \multicolumn{5}{|c|}{0.4} & \multicolumn{5}{|c|}{0.5} & \multicolumn{5}{|c|}{0.6} \\
\hline & $p_{1}$ & $p_{2}$ & $p_{3}$ & $|\delta|$ & $g(\delta)$ & $p_{1}$ & $p_{2}$ & $p_{3}$ & $\delta \mid$ & $g(\delta)$ & $p_{1}$ & $p_{2}$ & $p_{3}$ & $\delta$ & $g(\delta)$ \\
\hline$p_{A}$ & \multicolumn{5}{|c|}{ Type 1} & \multirow{2}{*}{\multicolumn{5}{|c|}{ Type 2}} & \multicolumn{5}{|c|}{ Type 3} \\
\hline 0.7 & .42 & .46 & .12 & .3 & .2 & .35 & & & & .2 & .28 & .54 & .18 & .1 & .2 \\
\hline 0.8 & .48 & .44 & .08 & .4 & .6 & .40 & .50 & .10 & .3 & .6 & .32 & .56 & .12 & .2 & .6 \\
\hline 0.9 & .54 & .42 & .04 & .5 & .2 & .45 & .50 & .05 & .4 & .2 & .36 & .58 & .06 & .3 & .2 \\
\hline
\end{tabular}

Table 5.2 $\overline{E \text { Loss }} / N G$ ( Design 1, Type 3 )

\begin{tabular}{cc|cccccccc}
\hline $\mathrm{d}$ & & 4 & 6 & 8 & 10 & 12 & 14 & 16 & 18 \\
\hline & 1 & 3.965 & 3.428 & 3.017 & 2.699 & 2.453 & 2.260 & 2.109 & $1.991^{*}$ \\
& 2 & $2.926^{*}$ & $2.723^{*}$ & $2.527^{*}$ & $2.357^{*}$ & $2.218^{*}$ & $2.112^{*}$ & $2.036^{*}$ & $1.991^{*}$ \\
& 3 & & 2.733 & 2.600 & 2.686 & 2.692 & 2.718 & 2.767 & 2.838 \\
& 4 & & & 3.600 & 3.769 & 3.962 & 4.167 & 4.374 & 4.565 \\
$c$ & 5 & & & & 5.137 & 5.535 & 5.938 & 6.303 & 6.567 \\
& 6 & & & & & 7.013 & 7.511 & 7.923 & $\mathbf{8 . 1 5 6}$ \\
& 7 & & & & & & 8.664 & $\mathbf{8 . 9 9 8}$ & 9.128 \\
& 8 & & & & & & & 9.588 & 9.623 \\
& 9 & & & & & & & & 9.844 \\
\hline
\end{tabular}

$(N=1000, M=30)$ The value with asterisk means optimum value of $\overline{E \operatorname{Loss}} / N G$ for each $d$

Table 5.3 $\overline{E \text { Loss }} / N G$ (Design 2, Type 3 )

\begin{tabular}{cc|cccccccc}
\hline \multicolumn{2}{c|}{} & 4 & 6 & 8 & 10 & 12 & 14 & 16 & 18 \\
\hline & 1 & 4.977 & 4.997 & 5.017 & 5.038 & 5.058 & 5.079 & 5.101 & 5.125 \\
& 2 & $2.468^{*}$ & 2.530 & 2.605 & 2.701 & $2.826^{*}$ & $2.996^{*}$ & $3.229^{*}$ & $3.559^{*}$ \\
& 3 & & $2.153^{*}$ & $2.378^{*}$ & $2.654^{*}$ & 2.995 & 3.419 & 3.952 & 4.625 \\
& 4 & & & 3.143 & 3.581 & 4.102 & 4.722 & 5.456 & 6.315 \\
$c$ & 5 & & & & 4.853 & 5.502 & 6.233 & 7.036 & 7.881 \\
& 6 & & & & & 6.897 & 7.618 & 8.335 & 8.992 \\
& 6 & & & & & & 8.681 & 9.207 & 9.613 \\
& 7 & & & & & & & 9.686 & 9.883 \\
& 8 & & & & & & & & 9.974 \\
\hline
\end{tabular}

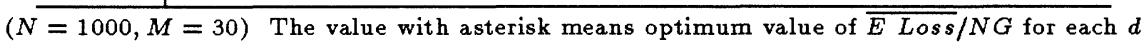

Table $5.4 \overline{\alpha_{i}}, E L^{*}$ and $c^{*}$ (Design 1)

\begin{tabular}{c|ccc|ccc|ccc}
\hline$p_{B}$ & \multicolumn{3}{|c|}{0.4} & \multicolumn{3}{c|}{0.5} & \multicolumn{3}{c}{0.6} \\
\hline & $\overline{\alpha_{1}}$ & $\overline{\alpha_{2}}$ & $\overline{\alpha_{3}}$ & $\overline{\alpha_{1}}$ & $\overline{\alpha_{2}}$ & $\overline{\alpha_{3}}$ & $\overline{\alpha_{1}}$ & $\overline{\alpha_{2}}$ & $\overline{\alpha_{3}}$ \\
\hline$p_{A}$ & \multicolumn{3}{|c|}{ Type 1 } & \multicolumn{3}{c|}{ Type 2 } & & Type 3 \\
0.7 & 0.957 & 0.038 & 0.005 & 0.844 & 0.125 & 0.031 & 0.618 & 0.261 & 0.121 \\
0.8 & 0.994 & 0.006 & 0.000 & 0.965 & 0.032 & 0.003 & 0.863 & 0.114 & 0.023 \\
0.9 & 1.000 & 0.000 & 0.000 & 0.997 & 0.002 & 0.001 & 0.980 & 0.019 & 0.001 \\
& $E L^{*}=1.164$ & $c^{*}=2$ & $E L^{*}=1.465$ & $c^{*}=2$ & $E L^{*}=1.991$ & $c^{*}=2$ \\
\hline \multicolumn{6}{c}{$(N=1000, M=30, d=18)$} &
\end{tabular}




\section{DOUKE}

Table $5.5 \overline{\alpha_{i}}, E L^{*}$ and $c^{*}$ ( Design 2)

\begin{tabular}{c|ccc|ccc|ccc}
\hline$p_{B}$ & \multicolumn{3}{|c|}{0.4} & \multicolumn{3}{c|}{0.5} & \multicolumn{3}{c}{0.6} \\
\hline & $\overline{\alpha_{1}}$ & $\overline{\alpha_{2}}$ & $\overline{\alpha_{3}}$ & $\overline{\alpha_{1}}$ & $\overline{\alpha_{2}}$ & $\overline{\alpha_{3}}$ & $\overline{\alpha_{1}}$ & $\overline{\alpha_{2}}$ & $\overline{\alpha_{3}}$ \\
\hline$p_{A}$ & \multicolumn{3}{|c|}{ Type 1 } & \multicolumn{3}{c|}{ Type 2 } \\
0.7 & 0.864 & 0.066 & 0.070 & 0.740 & 0.125 & 0.135 & 0.571 & 0.193 & 0.236 \\
0.8 & 0.946 & 0.028 & 0.026 & 0.872 & 0.073 & 0.055 & 0.743 & 0.153 & 0.104 \\
0.9 & 0.987 & 0.007 & 0.005 & 0.960 & 0.029 & 0.011 & 0.889 & 0.086 & 0.025 \\
& $E L^{*}=2.494$ & $c^{*}=2$ & $E L^{*}=3.207$ & $c^{*}=2$ & $E L^{*}=3.559$ & $c^{*}=2$ \\
\hline \multicolumn{1}{c}{$(N=1000, M=30, d=18)$}
\end{tabular}

Table 5.6 Comparison of three types on $\overline{\alpha_{1}}$ (Design 1)

\begin{tabular}{c|c|cccccccc}
\hline \multicolumn{2}{c|}{$M$} & 30 & 40 & 50 & 60 & 70 & 80 & 90 & 100 \\
\hline \multicolumn{2}{c|}{$\left(p_{A}, p_{B}\right)$} & & & & & & & & \\
\hline Type 1 & 0.70 .4 & 0.957 & 0.961 & 0.962 & 0.962 & 0.962 & 0.962 & 0.962 & 0.962 \\
& 0.80 .4 & 0.994 & 0.995 & 0.995 & 0.995 & 0.995 & 0.995 & 0.995 & 0.995 \\
& 0.90 .4 & 1.000 & 1.000 & 1.000 & 1.000 & 1.000 & 1.000 & 1.000 & 1.000 \\
\hline Type 2 & 0.70 .5 & 0.844 & 0.857 & 0.860 & 0.869 & 0.872 & 0.873 & 0.873 & 0.904 \\
& 0.80 .5 & 0.965 & 0.970 & 0.974 & 0.976 & 0.976 & 0.976 & 0.976 & 0.983 \\
& 0.90 .5 & 0.997 & 0.998 & 0.999 & 0.999 & 0.999 & 0.999 & 0.999 & 0.999 \\
\hline Type 3 & 0.70 .6 & 0.618 & 0.636 & 0.612 & 0.629 & 0.638 & 0.645 & 0.658 & 0.666 \\
& 0.80 .6 & 0.863 & 0.881 & 0.884 & 0.896 & 0.900 & 0.913 & 0.917 & 0.918 \\
& 0.90 .6 & 0.980 & 0.986 & 0.988 & 0.990 & 0.990 & 0.992 & 0.992 & 0.992 \\
\hline
\end{tabular}

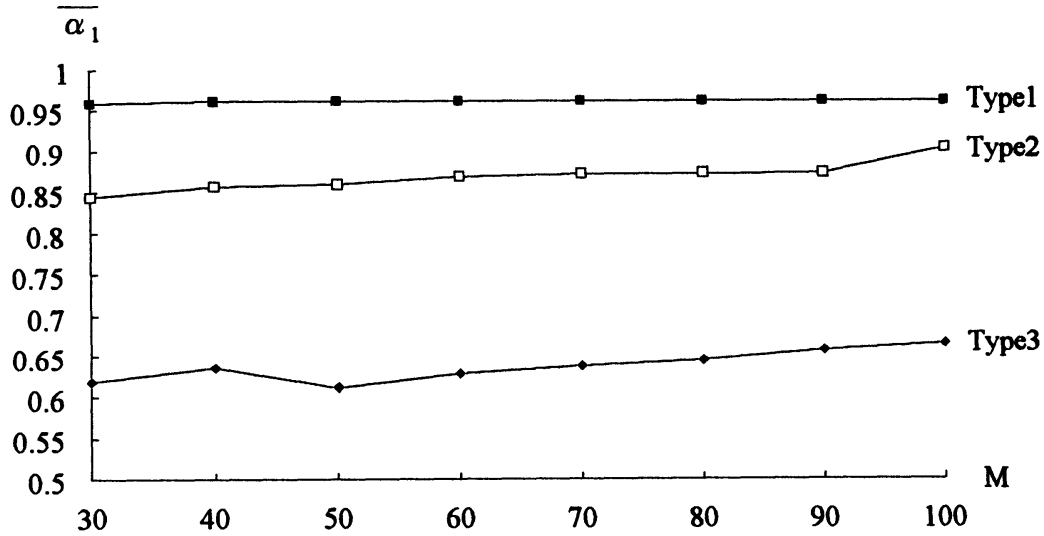

Figure 5.1 Comparison of three types on $\overline{\alpha_{1}}$ (Design 1)

$$
(N=1000, d=18)
$$

Table 5.7 Comparison of three types on $E L^{*}$ ( Design 1)

\begin{tabular}{c|c|cccccccc}
\hline \multicolumn{2}{c|}{$M$} & 30 & 40 & 50 & 60 & 70 & 80 & 90 & 100 \\
\hline Type 1 & $E L^{*}$ & 1.164 & 1.128 & 1.161 & 1.161 & 1.161 & 1.161 & 1.161 & 1.161 \\
& $c^{*}$ & 2 & 2 & 2 & 2 & 2 & 2 & 2 & 2 \\
Type 2 & $E L^{*}$ & 1.465 & 1.369 & 1.344 & 1.312 & 1.303 & 1.301 & 1.300 & 1.299 \\
& $c^{*}$ & 2 & 2 & 3 & 3 & 3 & 3 & 3 & 4 \\
Type 3 & $E L^{*}$ & 1.991 & 1.824 & 1.766 & 1.679 & 1.648 & 1.592 & 1.562 & 1.548 \\
& $c^{*}$ & 2 & 2 & 3 & 3 & 3 & 4 & 4 & 4 \\
\hline
\end{tabular}


On Sequential Design Based on Markov Chains

Table 5.8 Comparison of three types on $E L^{*}$ ( Design 2)

\begin{tabular}{c|c|cccccccc}
\hline \multicolumn{2}{c|}{$\mathrm{M}$} & 30 & 40 & 50 & 60 & 70 & 80 & 90 & 100 \\
\hline Type 1 & $E L^{*}$ & 2.494 & 1.472 & 1.282 & 1.197 & 1.179 & 1.175 & 1.174 & 1.174 \\
& $c^{*}$ & 2 & 3 & 4 & 4 & 4 & 4 & 4 & 4 \\
Type 2 & $E L^{*}$ & 3.207 & 1.950 & 1.504 & 1.262 & 1.173 & 1.141 & 1.129 & 1.118 \\
& $c^{*}$ & 3 & 3 & 3 & 4 & 4 & 4 & 4 & 5 \\
Type 3 & $E L^{*}$ & 3.559 & 2.536 & 1.910 & 1.619 & 1.405 & 1.397 & 1.233 & 1.175 \\
& $c^{*}$ & 2 & 3 & 3 & 4 & 4 & 4 & 5 & 5 \\
\hline \multicolumn{7}{c}{$(N=1000, d=18)$}
\end{tabular}

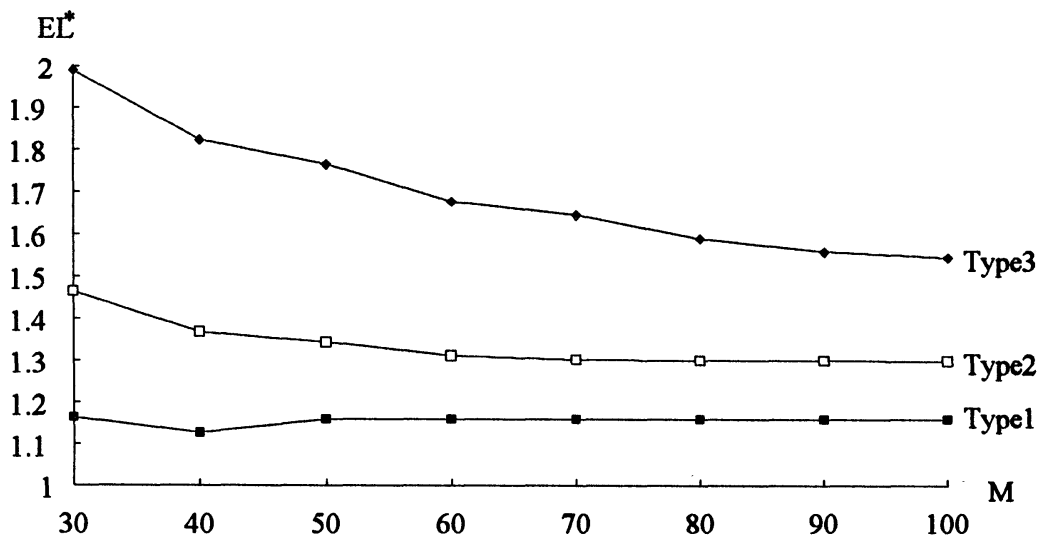

Figure 5.2 Comparison of three types on $E L^{*}$ ( Design 1)

$$
(N=1000, d=18)
$$

Table 5.9 Comparison of three types on $E L^{*}$ ( Design 1)

\begin{tabular}{c|c|ccccccccc}
\hline \multicolumn{2}{c|}{$\mathrm{N}$} & 1000 & 1500 & 2000 & 2500 & 3000 & 3500 & 4000 & 4500 & 5000 \\
\hline Type 1 & $E L^{*}$ & 1.164 & 1.274 & 1.384 & 1.494 & 1.604 & 1.713 & 1.824 & 1.932 & 2.044 \\
& $c^{*}$ & 2 & 2 & 2 & 2 & 2 & 2 & 2 & 2 & 2 \\
Type 2 & $E L^{*}$ & 1.465 & 1.830 & 2.195 & 2.560 & 2.925 & 3.290 & 3.655 & 4.020 & 4.385 \\
& $c^{*}$ & 2 & 2 & 2 & 2 & 2 & 2 & 2 & 2 & 2 \\
Type 3 & $E L^{*}$ & 1.991 & 2.751 & 3.510 & 4.270 & 5.030 & 5.790 & 6.750 & 7.310 & 8.069 \\
& $c^{*}$ & 2 & 2 & 2 & 2 & 2 & 2 & 2 & 2 & 2 \\
\hline
\end{tabular}

$(d=18, M=30)$

Table 5.10 Comparison of three types on $E L^{*}$ ( Design 2)

\begin{tabular}{c|c|ccccccccc}
\hline \multicolumn{2}{c|}{$\mathrm{N}$} & 1000 & 1500 & 2000 & 2500 & 3000 & 3500 & 4000 & 4500 & 5000 \\
\hline Type 1 & $E L^{*}$ & 2.494 & 3.332 & 4.170 & 5.048 & 5.847 & 6.685 & 7.523 & 8.361 & 9.199 \\
& $c^{*}$ & 2 & 2 & 2 & 2 & 2 & 2 & 2 & 2 & 2 \\
Type 2 & $E L^{*}$ & 3.207 & 4.529 & 5.850 & 7.171 & 8.493 & 9.814 & 11.135 & 12.456 & 13.778 \\
& $c^{*}$ & 2 & 2 & 2 & 2 & 2 & 2 & 2 & 2 & 2 \\
Type 3 & $E L^{*}$ & 3.559 & 5.181 & 6.803 & 8.426 & 10.048 & 11.670 & 13.292 & 14.915 & 18.537 \\
& $c^{*}$ & 2 & 2 & 2 & 2 & 2 & 2 & 2 & 2 & 2 \\
\hline \multicolumn{7}{c}{$(d=18, M=30)$}
\end{tabular}




\section{DOUKE}

\section{REFERENCES}

Armitage,P.(1957). Restricted Sequential Procedures, Biometrika, 44, 9-26.

Anderson,T.W.(1964). Sequential Analysis with Delayed Observations, Journal of the American Statistical Association, 59, 1006-1015.

Choi,S.C.(1968). Truncated Sequential Designs for Clinical Trials Based on Markov Chains, Biometrics, 24, 159-166.

Choi,S.C. and Clark,V.A.(1970). Sequential Decision for a Binomial Parameter with Delayed Observations, Biometrics, 26, 411-420.

Colton,T.(1963). A Model for Selecting One of Two Medical Treatments, Journal of the American Statistical Association, 58, 388-400.

Langenberg,P.and Srinivasan,R.(1981). On the Colton Model for Clinical Trials with Delayed Observations. Biometrics, 37, 143-148.

Sugimura,M., Goto,M. and Asano,C.(1969). Optimum Sequential Designs Based on Markov Chains for Selecting One of Tow Clinical Treatments. Faculty of Engineering, Kumamoto University, XV, 1-16.

Goto,M., Sugimura,M. and Asano,C.(1971). Numerical Tables of Optimum Sequential Designs Based on Markov Chains for Selecting One of Two Clinical Treatments. Bulletin of Mathematical Statistics,14, 27-56.

(Received August 1993; Revised December 1994) 